OUTP-00-08P

hep-th/0003074

March 2000

\title{
Brane Universe and Multigravity: Modification of gravity at large and small distances.
}

\author{
Ian I. Kogan] and Graham G. Ross [] \\ ${ }^{a}$ Theoretical Physics, Department of Physics, Oxford University \\ 1 Keble Road, Oxford, OX1 3NP, UK
}

\begin{abstract}
We consider a modification of gravity at large distances in a Brane Universe which was discussed recently [1] - [4]. In these models the modification of gravity at large distances is ultimately connected to existence of negative tension brane(s) and exponentially small tunneling factor. We discuss a general model which interpolates between Bi-gravity +-+ model [1] and GRS model [2]. We also discuss the possible mechanism of stabilization of negative tension branes in AdS background. Finally we show that extra degrees of freedom of massive gravitons do not lead to disastrous contradiction with General Relativity if the stabilization condition $\int d y \sqrt{-G^{(5)}}\left(T_{\mu}^{\mu}-2 T_{5}^{5}\right)=0$ [5] is implemented.
\end{abstract}

\footnotetext{
${ }^{1}$ i.kogan@physics.ox.ac.uk

${ }^{2}$ g.ross@physics.ox.ac.uk
} 
Recently, there has been considerable interest in theories in which the SM fields are localized on a 3-brane in a higher dimensional spacetime. The idea that a multidimensional Universe may not be of KK type, but rather a low dimensional $(3+1$ in the case of our Universe) brane dates back to early eighties when independently Akama [6] and Rubakov and Shapochnikov [7] suggested models of our Universe as topological defects and later Visser [8] and Squires [9] described how particles can be gravitationally trapped on the brane. The possibility that gravity be trapped on a brane was suggested by Gogberashvili 10

In papers of Antoniadis, Arkani-Hamed, Dimopoulos and Dvali [11, 12, 13] it was shown that the size of extra dimensions can be large, up to a $\mathrm{mm}$ scale. Then Randall and Sundrum proposed [14] a scenario in the geometry is non-factorizable and even infinitely large fifth dimension leads to the Newton law at macroscopic distances along the brane. In this picture the concept of a massless graviton localized on a positive tension brane was of utmost importance. Another important element was a negative tension brane.

Then in was suggested in [1] and later and independently in [2] that in some modification of RS scenario one can have even more strange behaviour - namely gravity is modified at both large and small scales. Even if explicit realizations of this idea suggested by Oxford group (KMPRS) [1] and Gregory, Rubakov and Sibiryakov (GRS) [2] are different one can see that they actually related to each other and share several key elements - for example the presence of free negative tension branes. There is another common feature in both models - exponential suppression and tunneling effect (in case of GRS model it was discussed by Csaki, Erlich and Hollowood in [3]). In this letter we demonstrate that these two models are actually two limiting cases of a more general model which we shall call +--+ or "multigravity" model and which interpolates between "bi-gravity" [1] and "quasi-localized" gravity [2], [3]. Besides this we are going to discuss the mechanism which will prevent negative brane destabilization - which is crucial for all these models. At the end we are going to discuss an important observation of Dvali, Gabadadze and Porrati 때 that in this models four-dimensional graviton is massive and this leads to discrepancy [15 between Newton law and light bending (and other experimentally observed post-Newtonian effects, for example Mercury perihelion precession). The remarkable resolution of this potentially disastrous discrepancy is that in a full theory there is actually a smooth limit for graviton mass $m \rightarrow 0$ if one take into account constraint $\int d y \sqrt{-G^{(5)}}\left(T_{\mu}^{\mu}-2 T_{5}^{5}\right)=0$ which is a necessary condition for stable compactification and correct cosmology on a brane [5]

Let us first discuss the KMPRS model [1] which consists of two positive branes located at the fixed points of a $S_{1} / Z_{2}$ orbifold with one negative brane which can move freely in between (see Fig. 目). It is easy to see that the two-brane RS model is nothing but the limiting case of our three-brane model, when the negative brane hits one of the positive branes.The model has two parameters, (at a fixed bulk curvature) - the warp factor and 


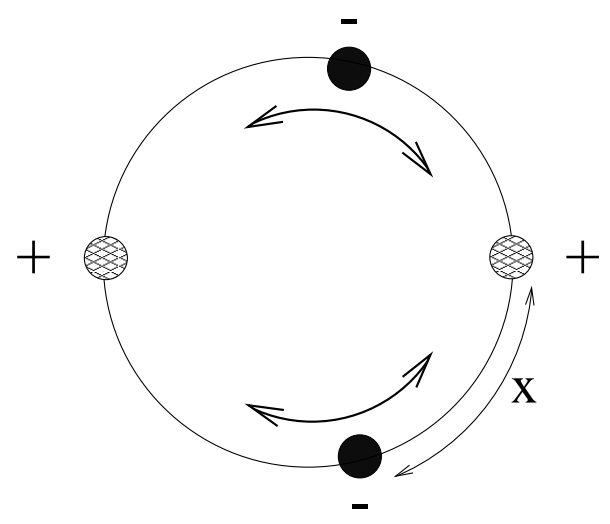

Figure 1: $\quad+-+$ model with two " + " branes at the fixed points and moving " - " branes. In the limiting case when $x \rightarrow 0$ we have a RS configuration.

the $x$ factor - which effectively measures the distance between one of the positive branes and the negative brane. The RS model corresponds to the limiting case $x=0$.

The GRS model can be obtained from this model by cutting negative brane in half, i.e. instead of one " - " brane with tension $-\Lambda$ one can take two branes with negative tension $-\Lambda / 2$ each and then move them apart, as on Fig. 2 If one then move the second " + " brane to infinity together with one of the " $-1 / 2^{\prime \prime}$ branes we shall get precisely the GRS picture - and the space between " $-1 / 2^{\prime \prime}$ brane is, of course, flat. Thus we have a new model - the +--+ model which depends on 3 parameters $-l_{L}$ and $l_{R}$ which are the differences between coordinates of left " $+{ }^{\prime \prime}$ and " $-1 / 2^{\prime \prime}$ branes and right " $+{ }^{\prime \prime}$ and " $-1 / 2^{\prime \prime}$ branes, and $l_{--}$ which is a difference between coordinates of the two " $-1 / 2^{\prime \prime}$ branes. This model consists of four parallel 3-branes in an $A d S_{5}$ space with cosmological constant $\Lambda<0$. The 5 -th dimension has the geometry of an orbifold and the branes are located at $y_{0}=0, y_{1}=l_{L}$ and $y_{2}=l_{L}+l_{--}$and $y_{3}=y_{2}+l_{R}$, where $y_{0}$ and $y_{2}$ are the orbifold fixed points (see Fig.2). Firstly we consider the branes having no matter on them in order to find a suitable vacuum solution. The action of this setup is:

$$
S=\int d^{4} x \int_{-y_{2}}^{y_{2}} d y \sqrt{-G}\left\{-\Lambda+2 M^{3} R\right\}-\sum_{i} \int_{y=y_{i}} d^{4} x V_{i} \sqrt{-\hat{G}^{(i)}}
$$

where $\hat{G}_{\mu \nu}^{(i)}$ is the induced metric on the branes and $V_{i}$ their tensions. Here we included negative $y$ and we are looking for solutions invariant with respect to $Z_{2}$ symmetry $y \rightarrow-y$. The notation is the same as in Ref. [14.

At this point we demand that our metric respects 4D Poincareé invariance. The metric

\footnotetext{
${ }^{3}$ The requirement that we have orbifold fixed points is not really necessary for our analysis, which is much more general
} 
ansatz with this property is the following:

$$
d s^{2}=e^{-2 \sigma(y)} \eta_{\mu \nu} d x^{\mu} d x^{\nu}+d y^{2}
$$

Here the "warp" function $\sigma(y)$ is essentially a conformal factor that rescales the $4 \mathrm{D}$ component of the metric. It satisfies the following differential equations:

$$
\left(\sigma^{\prime}\right)^{2}=k^{2}, \quad \sigma^{\prime \prime}=\sum_{i} \frac{V_{i}}{12 M^{3}} \delta\left(y-L_{i}\right)
$$

where $k=\sqrt{\frac{-\Lambda}{24 M^{3}}}$ is a measure of the curvature of the bulk. The brane tensions are tuned to $V_{0}=-\Lambda / k>0, V_{1}=V_{2}=\Lambda / 2 k<0, V_{2}=-\Lambda / k>0$. It is convenient to introduce 3 dimensionless parameters

$$
x_{L}=k l_{L}, \quad x_{R}=k l_{R}, \quad x_{-}=k l_{--}
$$

It is easy to see that when $x_{-}=0$ we have +-+ model [1] with the hierarchy fac-

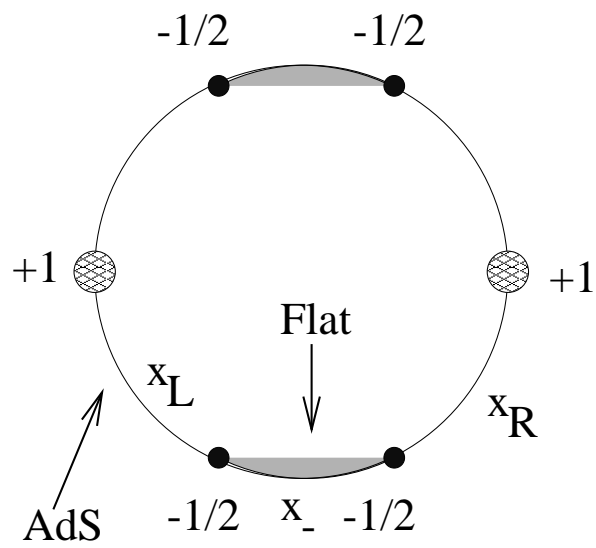

Figure 2: GRS model can be obtained from the left part of +--+ configuration. The whole configuration can be considered as two GRS models connected via flat space.

tor $w=e^{\left(x_{R}-x_{L}\right)}$ and parameter $x$ on Fig. 1 is $x_{R}$. In the limit of infinite $x_{-}$we have two infinitely separated GRS systems. Then it becomes quite clear that +--+ model smoothly interpolates between this two models. spectrum that follows from the dimensional reduction. Then we have to find the spectrum of (linear) fluctuations of the metric:

$$
d s^{2}=\left[e^{-2 \sigma(y)} \eta_{\mu \nu}+\frac{2}{M^{3 / 2}} h_{\mu \nu}(x, y)\right] d x^{\mu} d x^{\nu}+d y^{2}
$$

We expand the field $h_{\mu \nu}(x, y)$ in graviton and KK states plane waves:

$$
h_{\mu \nu}(x, y)=\sum_{n=0}^{\infty} h_{\mu \nu}^{(n)}(x) \Psi^{(n)}(y)
$$


where $\left(\partial_{\kappa} \partial^{\kappa}-m_{n}^{2}\right) h_{\mu \nu}^{(n)}=0$ and fix the gauge as $\partial^{\alpha} h_{\alpha \beta}^{(n)}=h_{\alpha}^{(n) \alpha}=0$. The function $\Psi^{(n)}(y)$ will obey a second order differential equation which after a change of variables reduces to an ordinary Schrödinger equation:

$$
\left\{-\frac{1}{2} \partial_{y}^{2}+V(y)\right\} \hat{\Psi}^{(n)}(y)=\frac{m_{n}^{2}}{2} \hat{\Psi}^{(n)}(y), \quad \hat{\Psi}^{(n)}(y) \equiv \Psi^{(n)}(y) e^{\sigma / 2}
$$

where potential $V(y)$ is determined by $\sigma(y)$. Qualitatively it is $\delta$-function potentials (attractive for " + " and repulsive for " $-"$ " branes) of different weight depending on brane tension and an extra smoothing term (due to the AdS geometry) that gives the potential a "volcano" form.

An interesting characteristic of this potential is that it always gives rise to a (massless) zero mode which reflects the fact that Lorentz invariance is preserved in $4 \mathrm{D}$ spacetime. This mode is normalizable for finite $x_{-}$and becomes non-normalizable in a GSR limit of infinite $x_{-}$. The interaction of the linearized gravitons to matter localized on a brane located at some $y$ is given by

$$
\mathcal{L}_{i n t}=\frac{f(y)}{M^{3 / 2}} \sum_{n \geq 0} \Psi^{(n)}(y) h_{\mu \nu}^{(n)}(x) T_{\mu \nu}(x)
$$

with $T_{\mu \nu}$ the energy momentum tensor of the SM Lagrangian and $f(y)$ is some universal function. From this expression the Newton potential on a brane is given by

$$
U(r) \sim \sum_{n \geq 0} \frac{\left(\Psi^{(n)}\right)^{2}(y)}{M^{3}} \frac{e^{-m r}}{r} \sim \int d m \frac{\Psi_{m}^{2}(y)}{M^{3}} \frac{e^{-m r}}{r}
$$

where $\rho(m)$ is the spectral density and it is discrete for $x_{-}=0$ and becomes continuous in a GRS limit of infinite $x_{-}$. We shall present full solution of this model for all $x_{-}$elsewhere [16.

Let us remind that one of the striking predictions of +-+ model is the fact that the first KK mode can be very light and strongly coupled compared to the rest of the KK states. This light mode can be so light that the corresponding wavelength can be by order of $1 \%$ of the observable size of the Universe while the second KK mode is in submillimeter region. Surprisingly enough this situation is not excluded experimentally and we called it "Bi-Gravity" - in all experimentally analyzed regions gravitational attraction is due to an exchange of two particles - the massless graviton and ultralight first KK mode. Only at scales larger than $10^{26} \mathrm{~cm}$ will the first KK mode decouple leading to a smaller gravitational coupling beyond this length scale. The reason the anomalously light KK mode exists is due to the fact that with more that one " + " brane there will be a bound state on each of them when they have infinite separation. At finite distances there is a mixing between the two localized states. One superposition is the true ground state while the other configuration 
has non-zero mass, but the gap may be very small - it is given by a tunneling factor. In the case $x>>1$ we obtained a reliable approximation to its mass

$$
m_{1}=2 k e^{-x_{L}-x_{R}}
$$

For $w=1$ we get $m_{1}=2 k e^{-2 x}$ and the wave functions $\Psi_{0}^{2}(0)=\Psi_{1}^{2}(0)$ with exponential accuracy. The masses of the other KK states in the above region are found to depend in a different way on the parameter $x$. The mass of the second state and the spacing $\Delta m$ between the subsequent states have the form:

$$
m_{2} \approx k e^{-x}, \quad \Delta m \approx \varepsilon k e^{-x}
$$

where $\varepsilon$ is a number between 1 and 2. Equations (10) and (11) show that, for large $x$, the lightest KK mode splits off from the remaining tower. This leads to an exotic possibility in which the lightest KK mode is the dominant source of Newtonian gravity!

Cavendish experiments and astronomical observations studying the motions of distant galaxies have put Newtonian gravity to test from submillimeter distances up to distances that correspond to $1 \%$ of the size of observable Universe, searching for violations of the weak equivalence principle and inverse square law. In the context of the graviton KK modes discussed above this constrains $m<10^{-31} \mathrm{eV}$ or $m>10^{-4} \mathrm{eV}$. Our exotic scheme corresponds to the choice $m_{1} \approx 10^{-31} \mathrm{eV}$ and $m_{2}>10^{-4} \mathrm{eV}$. In this case, for length scales less than $10^{26} \mathrm{~cm}$ gravity is generated by the exchange of both the massless graviton and the first $\mathrm{KK}$ mode ( $\mathrm{C}$ is some constant)

$$
U(r)=C \frac{\left(\Psi^{(0)}\right)^{2}(0)}{M^{3}}\left(\frac{1}{r}+\frac{\left(\Psi^{(1)}\right)^{2}(0)}{\left(\Psi^{(0)}\right)^{2}(0)} \frac{e^{-m_{1} r}}{r}\right)+O\left(e^{-m_{2} r}\right) \approx 2 C \frac{\left(\Psi^{0}\right)^{2}(0)}{M^{3}} \frac{1}{r}
$$

so gravitational constant is $G_{N}=2 C \frac{\left(\Psi^{0}\right)^{2}(0)}{M^{3}}$. According to this picture deviations from Newton's law will appear in the submillimeter regime $m_{2} r<1$ as the Yukawa corrections of the second and higher KK states become important. Also the presence of the ultralight first KK state will give deviations from Newton's law as we probe cosmological scales $m_{1} r>1$ (of the order of the observable universe) with $G_{N} / 2$ instead of $G_{N}$. The phenomenological signature of this scenario is that gravitational interactions will appear to become weaker (in general case by the factor $w$ [1]) for distances larger than $1 / m_{1} \sim 10^{26} \mathrm{~cm}$ !

The idea behind GRS construction was totally different - they did not have normalized modes, but rather continuous spectrum, however they have a "resonance" [3] which effectively was due to the fact that that the negative brane created a tunneling factor (negative brane acts as a repulsive potential) which effectively leads to a resonance in a wave function $\Psi_{m}(0)$ of gravitons

$$
\Psi_{m}^{2}(0)=\frac{c}{m^{2}+\Gamma^{2} / 4}+O\left(m^{4}\right)
$$



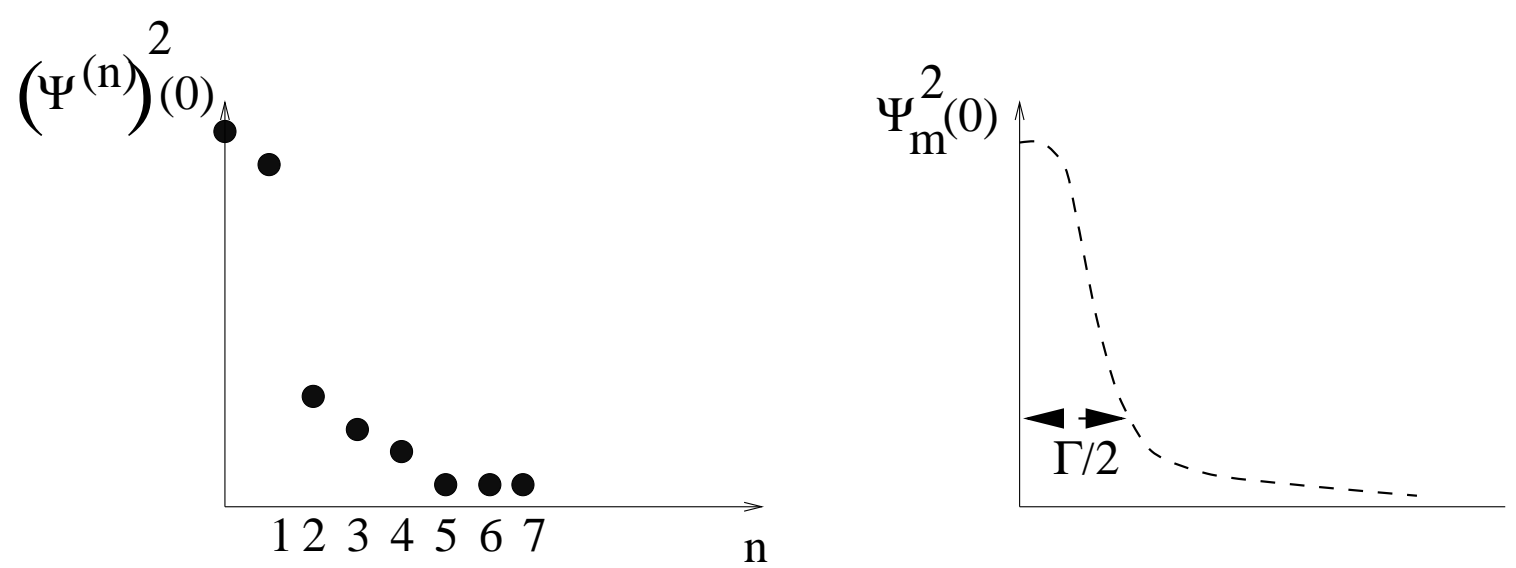

Figure 3: Behaviour $\Psi_{m}^{2}(0)$ in a +-+ model (discrete spectrum). For a general +--+ model the discrete spectrum density increases with the increase of $x_{-}$and spectrum becomes continuous in GRS limit.

From ([3]) one can see [2], [3]

$$
U(r)=\frac{2 c}{M^{3} \Gamma} \frac{1}{r} \int \frac{d x}{x^{2}+1} \exp \left(-\frac{\Gamma r}{2} x\right)
$$

and we see that for $r<<1 / \Gamma$ we recover the Newton $1 / r$ potential with

$$
G_{N}=\frac{\pi c}{M^{3} \Gamma}
$$

and for $r>1 / \Gamma$ potential is $1 / r^{2}$ and gravity is modified at large distances.

But this is just the same as we have on our discrete case (see Fig.3) - the reason is of course that $\Gamma \sim e^{-x}$. Thus we see that in both limits it the same tunneling factor $e^{-x}$ made modification of gravity possible - in discrete case it is exponentially small splitting between masses of massles graviton and first KK mode, in continuous case it is exponentially small virtual resonance width.

Of course one get the same behaviour in other models when positive branes are separated by negative branes. For example one may have a crystal [17] and in this case we have bands. Potential will be proportional to

$$
U(r) \sim \int d m \rho(m) \frac{e^{-m r}}{r}
$$

where $\rho(m)$ is band density and for narrow band we shall have multigravity again. All we need is that the width of the first band $\Gamma_{1}$ is much smaller than the separation between first and second bands (more details will be given in [16]). 

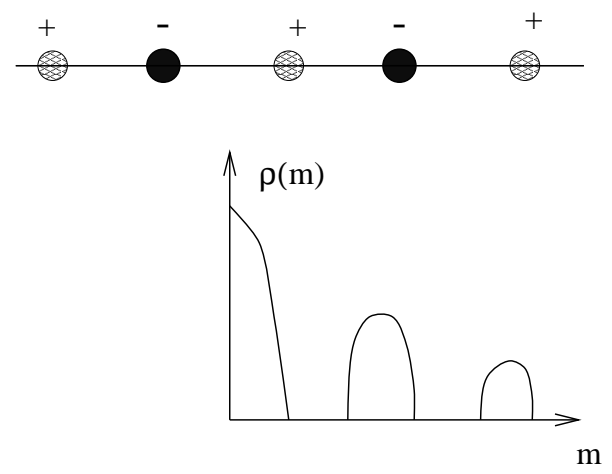

Figure 4: A periodic array of positive and negative branes is a model of Crystal Brane Universe [17].

Let's make some comments about the stability of the brane configuration discussed here. It involves a negative tension brane located between positive tension branes. Necessarily the negative tension (three) brane is free to move (in this configuration it cannot be located at a fixed point) and the question naturally arises about its stability. Being negative tension it will be advantageous to increase its volume and this may happen both by changing its location through the effects of the warp factor or through the brane bending. It is instructive to consider the first effect on its own by determining the effect of moving the position of the brane a distance $\delta$ from an initial position midway between the positive branes. In this case [1] the warp factor at the negative brane decreases by the factor $e^{-\kappa \delta}$, where $\kappa$ is the five dimensional curvature. Thus the volume of the negative brane decreases, implying the midpoint is a position of equilibrium for the "flat" brane. Consider now the effect of deforming the brane over some transverse size $l$ which may reasonable be taken to be the horizon size. The mean displacement of the brane about its equilibrium position is proportional to $R-R \cos \theta \sim R \theta^{2}=l \theta$, where $\theta=l / R \ll 1$. Thus the warp factor decreases by the factor $e^{-2 c \kappa l \theta}$ where $c$ is some constant. The deformed brane stretches by an amount $\approx\left(1+\theta^{2} / 2\right)$ in each transverse dimension so the net change in the brane volume is $\left(e^{-c \kappa l \theta}\left(1+\theta^{2} / 2\right)\right)^{3}$. Since the horizon size, $l$, will always be much larger than the Planck length $\approx \kappa^{-1}$ ), this naive argument suggests that the warp factor is the dominant effect and that the bending of the brane is energetically disfavoured. If this is true the brane configurations discussed in this paper may be stable structures and the modified gravitational interactions discussed above may indeed be possible .

Finally let us discuss problems raised in [四]. Contrary to massless four-dimensional graviton which has 2 polarizations the massive one has 5 possible polarizations. The tensor 
structure for graviton propagator is given by

$$
G^{\mu \nu, \alpha \beta}=C \frac{\left[1 / 2\left(g^{\mu \alpha} g^{\nu \beta}+g^{\nu \alpha} g^{\mu \beta}\right)-t g^{\mu \nu} g^{\alpha \beta}\right]+O\left(p p / p^{2}\right)}{p^{2}-m^{2}}
$$

where for all non-zero $m$ parameter $t=1 / 3$ [15] but for $m=0 t=1 / 2$. Then the onegraviton exchange amplitude for any two four-dimensional sources $T_{\mu \nu}^{1}$ and $T_{\alpha \beta}^{2}$ is given by

$$
T_{\mu \nu}^{1} G^{\mu \nu, \alpha \beta} T_{\alpha \beta}^{2}=C \frac{T_{\mu \nu}^{1} T^{2 \mu \nu}-t T^{1} T^{2}}{p^{2}-m^{2}}
$$

where $T=T_{\mu}^{\mu}$. Massive bodies at rest have only non-zero $T_{00}$ and $T=T_{00}$. Newton constant can be found from amplitude

$$
\frac{C(1-t)}{p^{2}-m^{2}} T_{00}^{1} T_{00}^{2}
$$

But there is another type of matter - light and for light $T=0$. The amplitude for light bending does not depend on $t$ and is given by

$$
\frac{C}{p^{2}-m^{2}} T_{00}^{1} T_{00}^{2}
$$

The ratio of two amplitudes is $1-t$. Because both amplitudes are known experimentally it is easy to find $t$-and of course it must be $t=1 / 2$ as in Einstein General relativity. If we take $t=1 / 3$ the ratio of two amplitudes is going to be $2 / 3$ instead of $1 / 2$ - and this is factor $4 / 3$ which was discussed in [4].

However this is not the whole story. We have a five-dimensional General relativity and the graviton propagator in five dimensions has tensor structure 18]

$$
G^{M N, P Q} \sim\left[1 / 2\left(g^{M P} g^{N Q}+g^{M Q} g^{N P}\right)-t g^{M N} g^{P Q}\right]+O\left(p p / p^{2}\right)
$$

where $t=1 / 3$ i.e. as massive four-dimensional graviton. The reason is very simple they both have 5 physical components. It is a well known fact in KK theory that after compactification the massless sector contains massless graviton, graviphotn and KK scalar - in total five degrees of freedom. At the same time massive KK excitations contain only massive $4 \mathrm{~d}$ graviton.

Let's then consider the full five-dimensional amplitude

$$
T_{M N} G^{M N, P Q} T_{P Q} \sim T_{M N}\left[1 / 2\left(g^{M P} g^{N Q}+g^{M Q} g^{N P}\right)-t g^{M N} g^{P Q}\right] T_{P Q}
$$

where $T_{M N}$ is a full stress-energy tensor. We don't have $T_{\mu 5}$ but besides $T_{\mu \nu}$ there is $T_{55}$. Then the total amplitude can be written as

$$
\begin{array}{r}
T_{\mu \nu} T^{\mu \nu}-t T T+(1-t) T_{5}^{5} T_{5}^{5}-2 t T T_{5}^{5}=\left[T_{\mu \nu} T^{\mu \nu}-\frac{1}{2} T T\right]+ \\
(1 / 2-t) T T+(1-t) T_{5}^{5} T_{5}^{5}-2 t T T_{5}^{5}
\end{array}
$$


The first line is the correct four-dimensional amplitude - all known experimental data gives this amplitude, so it will be nice to see that the second line is identically zero. Amusingly at $t=1 / 3$ the second line is a full square

$$
\frac{1}{6}\left(T-2 T_{5}^{5}\right)^{2}
$$

Why it is natural to have the condition $\left(T-2 T_{5}^{5}\right)=0$ ? First of all this is written in momentum representation. All amplitudes under consideration have zero momentum transfer in the fifth direction. This means that we have to integrate over the fifth coordinate $\int d y \sqrt{-G^{(5)}}\left(T-2 T_{5}^{5}\right)$. But this is precisely the integral condition which was discussed in [5]. The $g_{55}$ remains in equilibrium and the size of an extra dimension stays fixed if the stress energy of the 5D matter satisfies the following constraint $\mathrm{f}$ :

$$
\int d^{4} x d y \sqrt{-G^{(5)}}\left(\hat{T}_{\mu}^{\mu}-2 \hat{T}_{5}^{5}\right)=0
$$

To show what does this condition mean let us consider standard Kaluza-Klein decomposition

$$
G_{M N}^{(5)}=\left(\begin{array}{cc}
G_{\mu \nu}^{(4)} & 0 \\
0 & e^{2 \gamma}
\end{array}\right)
$$

and then consider the linear term

$$
\delta G_{M N}^{(5)} T^{M N}=\delta G_{\mu \nu}^{(4)} T^{\mu \nu}+\delta\left(e^{2 \gamma}\right) T^{55}
$$

in any system. Using $G_{\mu \nu}^{(4)}=e^{-\gamma} g_{\mu \nu}$, one can rewrite the above expression as

$$
\delta G_{M N}^{(5)} T^{M N}=e^{-\gamma} \delta g_{\mu \nu} T^{\mu \nu}-\delta \gamma\left(T-2 \hat{T}_{5}^{5}\right)
$$

Thus, we see that $T_{\mu \nu}$ is a source for the graviton while the combination $\left(T-2 T_{5}^{5}\right)$ is the source for the scalar (radion). In the presence of a mechanism that stabilizes the extra dimension (see for example [20] and [21] and references therein) the source for the constant mode of this scalar is identically zero. If we do not have a stabilization mechanism but, nevertheless, we look for solutions with a Newtonian limit, we still have to require the absence of the above term.

The conclusion is that there is a smooth limit $m \rightarrow 0$ in multigravity. The situation here similar to the Higgs mechanism. There is no smooth limit when the mass of a vector particle is given by hand - but there is a smooth limit when it emerges dynamically due to spontaneous symmetry breaking.

Acknowledgments: We would like to thank P. Kanti, S. Mouslopoulos, K.Olive, A. Papazoglou, M. Pospelov and M. Voloshin for interesting discussions. This work is supported

\footnotetext{
${ }^{4}$ This constraint was also derived from a topological argument in [19].
} 
in part by PPARC rolling grant PPA/G/O/1998/00567, the EC TMR grant FMRX-CT96-0090 and by the INTAS grant RFBR - 950567. One of us (I.I.K) would like to thank TPI, University of Minnesota for kind hospitality and stimulating discussions during visit in February 2000.

Note added in proof: During preparation of this paper for publications several

papers were published in which the $4 / 3$ problem [4] was discussed [22], but not the constraint (25).

\section{References}

[1] I. I. Kogan, S. Mouslopoulos, A. Papazoglou, G. G. Ross and J. Santiago, hepph/9912552

[2] R. Gregory, V. A. Rubakov and S. M. Sibiryakov, hep-th/0002072

[3] C. Csaki, J. Erlich and T. J. Hollowood, hep-th/0002161

[4] G. Dvali, G. Gabadadze, M. Porrati, hep-th/0002190

[5] P. Kanti, I.I. Kogan, K.A. Olive and M. Pospelov, hep- ph/9912266.

[6] K. Akama in Gauge Theory and Gravitation, Proceedings of the International Symposium, Nara, Japan, 1982, ed. K.Kikkawa, N.Nakanishi and H. Nariai (Springer-Verlag, 1983), 267; e-version is: K.Akama, hep-th/0001113

[7] V.A.Rubakov and M.E. Shaposhnikov, Phys. Lett. B125 (1983) 136.

[8] M. Visser, Phys. Lett. B159 (1985) 22.

[9] E. J. Squires, Phys. Lett. B167 (1985) 286.

[10] M. Gogberashvili, hep-ph/9904383

[11] N. Arkani-Hamed, S. Dimopoulos and G. Dvali, Phys. Lett. B429 (1998) 263.

[12] N. Arkani-Hamed, S. Dimopoulos and G. Dvali, Phys. Rev. D59 (1999) 086004.

[13] I. Antoniadis, N. Arkani-Hamed, S. Dimopoulos and G. Dvali, Phys. Lett. B436 (1998) 257.

[14] L. Randall and R. Sundrum, Phys. Rev. Lett. 83 (1999) 3370, Phys. Rev. Lett. 83 (1999) 4690; hep-th/9905221,9906064. 
[15] H. van Dam, M.Veltman, Nucl.Phys. B22, (1970) 397;

V.I. Zakharov, JETP. Lett. 12 (1970), 312.

[16] I. I. Kogan, S. Mouslopoulos, A. Papazoglou, G. G. Ross, to be published

[17] N. Kaloper, hep-th/9912125

[18] "Modern Kaluza-Klein Theories", edited by T. Appelquist, A. Chodos and P.G.O. Freund, Addison-Wesley, (1987)

[19] U. Ellwanger, hep-th/9909103.

[20] W.D. Goldberger and M.B. Wise, Phys. Rev. D60 (1999) 107505; hep-ph/9907447; hep-ph/9911457.

[21] C. Csáki, M. Graesser, L. Randall, and J. Terning, hep-ph/9911406.

[22] C. Csaki, J. Erlich and T. J. Hollowood, hep-th/0003020;

R. Gregory, V. A. Rubakov and S. M. Sibiryakov, hep-th/0003045

G. Dvali, G. Gabadadze, M. Porrati, hep-th/0003054 